\title{
Le conflit dans « l'entente ». Coopération et compétition dans les associations paysannes de Koungheul (Sénégal)
}

\section{Giorgio Blundo}

\author{
(2) OpenEdition \\ Journals \\ Édition électronique \\ URL : http://journals.openedition.org/apad/3773 \\ DOI : 10.4000/apad.3773 \\ ISSN : 1950-6929 \\ Éditeur \\ LIT Verlag \\ Édition imprimée \\ Date de publication : 1 décembre 1992 \\ Référence électronique \\ Giorgio Blundo, «Le conflit dans « l'entente ». Coopération et compétition dans les associations \\ paysannes de Koungheul (Sénégal) », Bulletin de l'APAD [En ligne], 4 | 1992, mis en ligne le 26 juin 2008, \\ consulté le 07 septembre 2020. URL : http://journals.openedition.org/apad/3773 ; DOI : https:// \\ doi.org/10.4000/apad.3773
}

Ce document a été généré automatiquement le 7 septembre 2020

Bulletin de I'APAD 


\title{
Le conflit dans « l'entente ». Coopération et compétition dans les associations paysannes de Koungheul (Sénégal)
}

\author{
Giorgio Blundo
}

Su fukk di gas, fukk di suul, pëndaay bari, waaye pax du fa am.

"Si dix personnes se mettent à combler le trou que dix autres creusent, on aura

beaucoup de poussière mais pas un trou" (proverbe wolof) ${ }^{1}$

Lorsque l'on étudie les organisations paysannes en Afrique, on avance sur un terrain particulièrement insidieux et glissant, où le discours devient normatif et donne l'illusion de suffire, à lui seul, à modifier la réalité sociale. Il est indéniable que les expériences multiples et multiformes d'organisation populaire sont actuellement sous les projecteurs des opérateurs de développement et des chercheurs en sciences sociales.

2 Pour les uns, l'intérêt se justifie par la nécessité de repenser les politiques de développement, en partant du constat que celles jusqu'ici proposées par les Etats et les bailleurs de fonds internationaux ont abouti à l'échec parce qu'elles ne correspondaient pas aux projets et aux desiderata des populations. L'attention s'est désormais portée sur les processus d'auto-promotion, où "ce sont les acteurs de base qui définissent leurs objectifs et finalités, et déploient des stratégies propres intégrant les relations avec les autres acteurs" (Kwan Kai Hong, 1991 : 25).

Pour les autres, s'ouvre un vaste domaine d'investigation et de réflexion, dont la diversité des perspectives était bien représentée dans ce colloque.

Objet de "convoitise" pour développeurs et chercheurs, les associations paysannes, et le débat qui les concerne, se situent ainsi, comme le dit Olivier de Sardan, à "l'intersection entre sciences sociales et pratiques de développement", un lieu privilégié "d'éclosion d'attitudes populistes" (1990: 475) et en particulier de celles que le même auteur définit comme "populisme idéologique" (op. cil. : 482-83). 
5 En effet, le danger est réel - et les exemples ne manquent pas - de donner une interprétation des formes d'organisation paysanne qui soit tributaire du stéréotype de la "communauté traditionnelle". Celle-ci serait une entité simple, homogène, durable et autarcique, caractérisée par une faible différenciation sociale, par une homogénéité d'objectifs et d'intérêts et par l'absence de conflits, le tout baignant dans l'a-temporalité.

6 Ainsi, l'image des groupements paysans est parfois associée, à tort ou à raison, aux pratiques villageoises ancestrales fondées sur l'entraide, la solidarité et la réciprocité. On affirme plus précisément que les anciennes institutions ont souvent fourni le "matériel culturel" pour bâtir les associations modernes consacrées au développement endogène du village.

7 Mais il serait simpliste de penser que les groupements sont des lieux de cohésion, seulement en vertu de l'activation dans ces structures "modernes" des prétendues règles traditionnelles de la coopération et de la solidarité villageoise. Mes recherches au Sénégal ${ }^{2} \mathrm{~m}$ 'ont permis de constater que persistent certaines pratiques d'entraide traditionnelles, mais que les groupements paysans constitués pour le "développement" sont des institutions autres, qui fonctionnent selon des logiques différentes et qui sont de ce fait sources et lieux de dynamiques nouvelles, souvent fort conflictuelles.

8 Ainsi, l'étude de cas que je propose vise à montrer qu'autonomie locale et développement endogène ne vont pas forcément de pair avec cohésion et harmonie.

9 Je veux mettre l'accent sur les associations paysannes comme lieux de rencontre et de confrontation d'acteurs sociaux différents ayant tous leurs intérêts et stratégies, et montrer comment cette confrontation peut parfois faire remonter à la surface des conflits latents, ou être la source d'antagonismes nouveaux.

10 A cet effet, je propose une approche des associations par l'analyse des conflits, des antagonismes et des clivages en leur sein. Ce choix m'a été suggéré par mes interlocuteurs durant le séjour sur le terrain. Dès le début, en effet, j'ai été frappé par la contradiction manifeste entre l'évocation des valeurs de cohésion et d'entraide sans cesse réaffirmée par les leaders aux membres des groupements, et constituant également une carte de visite à l'égard des interlocuteurs externes, et le fonctionnement réel des groupes, perturbé par les désaccords, les conflits d'intérêts et les négociations longues et difficiles.

11 Certes, rappeler que le, conflit est endémique au sein de la réalité sociale relève d'un truisme, puisque de l'Ecole de Manchester à l'anthropologie dynamique de Balandier, en passant par les études de Leach et les théories de l'action, voilà 40 ans que la problématique du conflit a été introduite dans les analyses des anthropologues.

12 Cependant, il me semble que ce changement de perspective, des "structures" aux "processus", reste encore inachevé dans les réflexions qui animent les pratiques de développement, surtout dans le domaine de la "participation paysanne".

13 Analyser les dynamiques conflictuelles au sein des organisations paysannes s'avère intéressant à deux niveaux :

14 1) celui du repérage, sur le terrain, des acteurs sociaux individuels et collectifs et de leurs alliances ;

15 2) celui de la réflexion socio-anthropologique sur la nature des groupements étudiés. 
16 A travers l'analyse de quelques cas de crise et de conflit, je vais tenter de décrire la composition sociale, l'organisation, les objectifs et leurs modalités de réalisation, les formes de leadership et de participation des associations paysannes ainsi que leur degré d'intégration dans la vie sociale du village. Apparaitront ainsi les contradictions internes aux associations et les divers enjeux qui découlent de leur implantation dans la société locale.

17 Après avoir esquissé une présentation de la zone de Koungheul et des traits saillants des associations paysannes étudiées, j'aborderai le thème central de cette contribution. Des groupements "Karikas" à l'Entente des groupements du COMI (1982-92)

18 Mon étude concerne des associations paysannes de l'arrondissement de Maka Yop (ex-arrondissement de Koungheul, région de Kaolack), correspondant au secteur oriental du bassin arachidier sénégalais.

19 Le climat de la zone de Koungheul est de type sahélo-soudanais. La pluviométrie moyenne a été de $630 \mathrm{~mm}$. pour la période allant de 1981 à $1990^{3}$.

20 Le système agraire se fonde sur deux cultures principales: l'arachide, culture commerciale, et les céréales, culture vivrière. A celles-ci s'ajoutent le coton, le manioc et un peu de maraîchage dans les bas-fonds. L'arachide reste toutefois la culture dominante, bien que l'on doive signaler une diminution récente de son exploitation, en réaction à la Nouvelle Politique Agricole (NP A), qui a entre autre supprimé le crédit d'équipement agricole pour l'arachide et réduit le nombre des points de collecte du produit. L'agriculture pratiquée est de type extensif, malgré la progressive disparition de la pratique de la jachère.

21 Les associations paysannes appartiennent à cinq villages (Fass Ndjebel, Kasassa, Keur Ngaye, Ndoune et Nioro Thialène) situés dans un rayon d'environ $30 \mathrm{~km}$ de Koungheul. Leur population, en majorité wolof, varie entre 181 (Kasassa) et 864 habitants (Ndoune).

22 Les cinq associations étudiées naissent en 1982. A cette époque, elles font partie d'un ensemble d'une trentaine de groupements nés sous l'impulsion d'un paysan de la zone de Koungheul qui s'était improvisé promoteur d'actions de développement.

Ce personnage va jouer un rôle d'intermédiaire entre un organisme d'appui (Caritas-Kaolack) et ces organisations naissantes. Après avoir poussé ces dernières à s'adonner au maraîchage principalement pour attirer des bailleurs de fonds, il parvient à escroquer 500.000 FCFA à chaque groupement. Cette somme, versée à Caritas, aurait donné droit, selon ses dires, à un forage ou à des moulins à mil. Une fois la supercherie découverte et l'argent perdu, la plupart des groupements "Karikas" ${ }^{4}$ se dissolvent, mais un groupe restreint (formé par les associations objet de notre étude) décide de continuer et demande en 1984 une aide à la mission catholique de Koungheul.

24 Le partenariat avec la mission dure de 1984 à 1986. Durant cette période, la mission finance la réparation de quelques puits et, dans les mois de "soudure", distribue du mil et du riz par l'intermédiaire des associations.

En 1986, appelée par la mission, une ONG italienne, la COMI, vient en appui des associations. L'approche de l'intervenant est fondée sur l'aide à la constitution des micro-réalisations effectuées avec la collaboration de volontaires expatriés. Cette action donne un second souffle aux groupements et couronne leur rêve d'être encadrés par un véritable projet. A l'arrivée de l'ONG, les associations ont déjà la physionomie actuelle de petits groupes: leurs effectifs varient entre 28 (Kasassa) et 51 membres (Ndoune). Avant la crise de 1984, la nouveauté des activités et les promesses de 
l'animateur avaient galvanisé la quasi-totalité des villageois: c'est le cas des associations de Ndoune et Keur Ngaye, qui comptaient 373 et 270 membres respectivement.

Nées autour de petits jardins potagers créés essentiellement pour attirer les bailleurs de fonds, les associations ont diversifié leurs activités au fil des années. Outre les activités agricoles (maraîchage de saison sèche, reboisement, arboriculture, riziculture de bas-fond et champs communautaires de céréales et arachides durant l'hivernage), elles s'occupent de la lutte anti-érosive, de l'aménagement des bas-fonds et de la constitution de caisses d'épargne ainsi que de banques céréalières et semencières. Le programme d'appui de l'ONG prend en charge le volet formation (techniques agricoles, alphabétisation, comptabilité).

En 1992, les associations des villages de Fass Ndjebel, Kasassa, Kër Ngaye et Ndoune ont constitué, avec quatre autres associations de la zone (dans les villages de Kër Ablaye, Jam Jam Koukoto, Kër Goury et Sinthiou Pathe), l'Entente des Groupements du COMI, ayant le statut juridique de GIE ${ }^{5}$. Comme au tout début, cette formation, qui est le fruit de longues négociations entre les groupements, a été marquée par la perte des cotisations destinées aux démarches administratives, détournées par un animateur local qui s'était chargé de la chose.

Il faut remarquer en premier lieu que ces associations volontaires mixtes sont composées principalement par des femmes (celles-ci représentent 70\% de l'ensemble des effectifs).

De plus, les associations sont investies par une population adulte occupant les niveaux les plus élevés de la hiérarchie familiale. En effet, parmi les hommes mariés (87,2\% de l'ensemble des effectifs masculins), $88 \%$ d'entre eux sont des borom kër (chef d'une unité de résidence) et des borom njël (chef de ménage exploitant) et seulement $12 \%$ sont des surga boromjabaar ou botale (chef de ménage dépendant).

Il en va de même pour la partie féminine : $80 \%$ des femmes membres des associations sont mariées, tandis que $11 \%$ sont des femmes âgées, veuves ou divorcées, résidant dans le foyer d'un de leurs enfants, et seul $9 \%$ du total sont des adolescentes non-mariées. En outre, les deux tiers des 85 femmes mariées appartiennent à un ménage polygamique, et plus de la moitié d'entre elles jouissent du statut de première épouse (awo). En effet, comme la participation aux associations comporte des activités qui vont s'ajouter aux tâches que chaque femme doit effectuer à l'intérieur de l'organisation productive du kër, elle est l'apanage des femmes ayant des co-épouses ou qui ont, pour des raisons tenant à leur âge, abandonné leur rôle de productrices dans le foyer.

31 Toutefois, l'homogénéité du statut socio-familial cache des différences marquées quant à l'appartenance aux classes d'âge : $67 \%$ des femmes mariées ont entre 21 et 45 ans, mais les hommes ont plus de 45 ans dans 53\% des cas. Dans ce groupe, la tranche d'âge au-dessous de 30 ans est fort peu représentée.

Les responsables justifient l'absence des "cadets" par le fait qu'ils n'ont pas envie de travailler et préfèrent passer la saison sèche à "gaspiller" l'argent gagné lors de la traite de l'arachide.

33 Mais, au-delà des explications fournies, les associations reproduisent la hiérarchie existant à l'intérieur de la famille élargie. En particulier, la coexistence de deux groupes sociaux - les aînés et les cadets - traditionnellement en compétition (voir Diop, 1985 : 
177-181) serait assez problématique. En outre, les principales activités productives des groupements se déroulent durant la saison sèche, période de l'année où les jeunes célibataires vont à la recherche de revenus additionnels en vue du mariage. A cet effet, leurs groupes d'âge traditionnels (mbotaay $u$ waxambaane yi) réduisent à cette époque leurs activités à des réunions ayant un caractère essentiellement ludique ${ }^{6}$. Or, les groupements paysans étudiés s'adonnent à des activités généralement peu rentables pour chaque membre (c'est le cas du maraîchage, dont les produits sont presque entièrement commercialisés en vue de la constitution d'un fonds commun pour des réalisations collectives) ou ne l'étant qu'à long terme (reboisement, protection de l'environnement, formation), donc peu intéressantes pour les jeunes d'un point de vue économique. Ainsi, les rares fois où les organismes d'appui ont essayé de faire collaborer les groupements d'adultes et les mbootaay des waxambaane, cela s'est traduit par un échec.

Ces associations se sont formées à travers trois canaux principaux: les rapports de voisinage et d'amitié, les relations de parenté et d'alliance, ainsi que les relations de clientèle.

La participation du kër (groupe local de résidence) à la vie associative varie selon les villages et les familles. A l'échelle des cinq villages, 57,2\% des kër impliqués dans l'associationnisme le sont à travers deux ou plus de leurs membres. Généralement, il s'agit du chef de famille avec sa/ses femme(s), et d'éventuels enfants, parfois mariés. Il est très rare qu'un homme participe à titre individuel au groupement. En effet, les membres ayant adhéré à titre personnel et en dehors des réseaux de parenté sont dans $86,7 \%$ des cas de sexe féminin.

Les liens de parenté ont une importance relative pour les femmes et leur solidarité, très importante, s'exprime dans plusieurs formes associatives extra-parentales: groupes d'âge d'adolescentes et de jeunes mariées (mbootaay u janxa yi et mbootaay u jéég ji), tontines, ndey dike (voir Blundo, 1990).

37 Les hommes, en revanche, ne participent aux groupes d'âge que jusqu'à l'accès au statut de chef de ménage exploitant (borom njël). S'associer entre parents est probablement une stratégie que le groupe masculin adopte pour éviter ou minimiser les risques d'éclatement de conflits d'intérêt et d'autorité.

$38 \mathrm{Au}$ vu de cette présentation, un tableau provisoire se dessine: celui d'associations composées d'une population relativement homogène, occupant des positions statutaires assez proches, et liée principalement par des relations de parenté et d'affinité.

39 Toutefois, il ne faut pas conclure hâtivement à un fonctionnement harmonieux des associations: tout d'abord, n'oublions pas que ces dernières ont un caractère volontaire, ce qui implique que chaque membre a adhéré aussi par intérêt personnel.

Ensuite, le clivage hommes/femmes et dirigeants/membres divise les associations et conditionne profondément leur dynamique et leur organisation.

Contradictions et conflits au sein des associationsLe jardin, lieu de rencontre entre groupes sociaux différents

41 Dans mon analyse, je distingue entre conflits internes, qui opposent les membres d'un groupement, et conflits externes, qui opposent les associations à d'autres acteurs sociaux, individuels ou collectifs, à l'échelle villageoise. Cette distinction reste cependant indicative, étant donné qu'un même conflit peut activer des systèmes 
complexes de relation et d'alliance et peut dès lors déclencher des antagonismes à d'autres niveaux.

Le jardin maraîcher et potager est toujours la première étape réservée au visiteur des associations: gouverneurs, responsables d'ONG, ambassadeurs, tous passent par le jardin. C'est pourquoi je commencerai aussi par là, dans cette introduction aux dynamiques internes des associations de la zone de Koungheul.

Le jardin, qui pour l'observateur de passage représente l'expression du communautarisme villageois, est en revanche un lieu de conflits latents entre "groupes stratégiques" (Bierschenk, 1988) ayant des intérêts contradictoires et étant tributaires de négociations continues : les hommes-responsables et les femmes-productrices.

Je vais le montrer à travers le cas de Nioro Thialène :

Durant la campagne horticole 1986-87, le bénéfice obtenu du jardin est entièrement détourné par le trésorier. Sa dénonciation aux autorités et le remboursement de la somme ne suffisent pas à empêcher la défection de bon nombre de participants : l'association se réduit de 30 à 17 membres. Le noyau qui reste se trouve confronté au dilemme de l'emploi de l'argent, et le conflit éclate: si les femmes veulent partager les bénéfices entre chaque membre, les hommes ont décidé que cet argent devrait cofinancer un puits que l'ONG d'appui est prête à réaliser. La dispute s'étend à tout le village: les maris des participantes, restés en dehors de l'association, interviennent en accusant le chef du village - qui joue aussi le rôle de leader associatif - de vouloir exploiter leurs épouses. Sous la menace d'abandon formulée par les femmes, les responsables décident alors de partager l'argent de la caisse. Ceux qui souhaitent alors rester dans l'association sont tenus de reverser la somme au trésorier. Mais l'année suivante, le groupe ne comptera plus que quatre hommes et quatre femmes, et se désagrégera au bout de quelques mois.

Il faut remarquer tout d'abord que ces associations paysannes "modernes" rassemblent des groupes sociaux - les hommes et les femmes - qui sont normalement séparés dans les modèles associatifs wolof traditionnels. Les conflits inhérents à cette nouvelle forme d'activité commune demeurent à l'état latent si chaque groupe social sent que ses intérêts et objectifs sont satisfaits ou du moins le seront à court ou moyen terme. L'éclatement de l'association de Nioro Thialène se comprend mieux si nous considérons que les responsables n'ont pas su garder un équilibre entre la logique des hommes, la poursuite d'un bien collectif et non-exclusif (le puits), et la logique des femmes, visant la satisfaction d'intérêts individuels.

En effet, chaque groupe social n'a pas les mêmes attitudes par rapport au maraîchage :

- pour les hommes, qui ont fondé les associations, le jardin n'est pas seulement important en soi, comme moyen de diversification du régime alimentaire et comme fournisseur de revenus d'appoint. Il est, depuis l'origine des groupements, l'aimant qui attire les bailleurs de fonds en tant que symbole des efforts collectifs des paysans. La preuve en est que le maraîchage a connu une involution une fois les puits creusés. Depuis 1986, les surfaces cultivées n'ont pas véritablement augmenté. L'abandon du maraîchage de la part des hommes est dû aussi à des charges de travail importantes et contraignantes en emploi du temps par rapport aux bénéfices escomptés en termes monétaires ${ }^{7}$.

- Les femmes jugent en revanche le maraîchage très utile, parce que cela leur permet de diversifier leurs revenus et d'utiliser les légumes dans la sauce qu'elles doivent préparer quotidiennement. En outre, les femmes sont indispensables à la formation des groupes, et cela pour deux raisons : elles sont l'élément-clef de tout discours populiste qui se respecte, gouvernemental et non-gouvernemental, et leur présence légitime donc l'existence de 
l'association. De plus, elles constituent une force de travail non négligeable pour des hommes qui ne veulent pas "gaspiller toute la saison sèche au milieu des tomates et des choux pommés".

Cela étant, toutes les associations ont élaboré un système d'exploitation maraîchère individuel-collectif (à l'exception de celle de Nioro Thialène, ce qui explique en partie le conflit qui a été à la base de son éclatement). Le jardin ${ }^{8}$, clôturé et situé à proximité d'un puits, est divisé en deux secteurs, l'un communautaire (tolu mbootaay) et l'autre destiné à la production individuelle, cultivé principalement par les femmes (tolu jigeen). Les produits du tolu mbootaay sont entièrement commercialisés, et les bénéfices sont recueillis dans un fonds commun devant financer d'autres activités du groupement, tandis que ceux des parcelles individuelles sont à la fois auto-consommés et commercialisés. Ces deux secteurs jouissent de structures et de moyens communs, ce qui détermine l'interaction entre les deux groupes, l'organisation du travail et la répartition des tâches. Les hommes s'occupent des travaux d'implantation du jardin, de l'entretien du champ communautaire et du pompage de l'eau en cas de présence de systèmes d'exhaure à traction animale. Le semis en pépinière et le repiquage sont effectués collectivement par les deux groupes. Les femmes se chargent de l'irrigation et, dans le cas de puits non-équipés, du puisage de l'eau.

Mais l'accès aux rôles directifs reste limité au groupe des hommes, qui contrôle ainsi le fonctionnement et les décisions des associations malgré sa représentation minoritaire. Les femmes sont représentées par leur leader (ndey u jigeen), une femme âgée qui les organise et prend la parole lors des réunions. Les décisions et les orientations sont prises en principe à ces occasions, où les membres sont invités à s'exprimer librement. En réalité, dans ces assemblées, est réaffirmée la hiérarchie sociale existant dans les associations. Si l'on observe qui prend la parole et comment les places assises sont distribuées, on s'aperçoit que les femmes se disposent toujours derrière les hommes. Souvent elles tournent même le dos à l'assemblée, paraissant affecter un désintérêt à l'égard de ce qui se passe. La discussion, parfois animée, semble concerner les hommes seulement, et les éventuelles décisions sont alors ratifiées par le leader des femmes.

Jusqu'à présent, la menace de quitter le groupement est pour les femmes la seule forme de pression qu'elles peuvent utiliser avec un certain succès, comme nous venons de le voir.

L'organisation et le contrôle, sources de dynamiques conflictuelles

50 Les activités collectives des associations ne se limitent pas au maraîchage, mais incluent le reboisement, l'arboriculture, l'aménagement des bas-fonds et la lutte anti-érosive. Aux travaux manuels s'ajoute aussi la participation à des réunions périodiques et à des stages de formation, bien que ces derniers concernent quelques représentants seulement.

51 Les associations paysannes "modernes" demandent donc un engagement personnel qui dépasse de loin, en durée et en complexité, celui requis par les travaux collectifs traditionnels. Pour gérer au mieux cet ensemble d'activités, chaque association s'est dotée de règlements internes de complexité et d'efficacité inégales.

52 Le contrôle dans les associations se base sur un système d'amendes en cas d'absence aux réunions ou aux travaux collectifs, et de sanctions en cas de fautes plus graves. Mais les règlements sont rarement appliqués, ce qui augmente les conflits latents. Je ne peux pas rendre compte ici des nombreuses disputes concernant l'organisation, ni des 
ajustements qui ont suivi. Il s'agit d'un problème commun aux cinq associations. Certaines contradictions de fond dans leur organisation et dans leur composition sociale l'expliquant :

- en premier lieu, l'organisation mixte des groupements. Payer une amende se traduit comme un aveu public de la faute. Parfois, ce sont même des femmes qui sont chargées de la perception des amendes et un homme, surtout s'il est un aîné, préférera quitter le groupe plutôt que de s'humilier devant elles,

- ensuite, les associations étant composées en bonne partie par des parents, leurs membres se trouvent confrontés à des conflits de loyauté : se ranger aux côtés du parent fautif ou accepter les règles associatives ? La contradiction entre la logique associative extra-parentale et la logique lignagère s'est manifestée à plusieurs reprises,

- enfin, le fait que la majorité des fautes concerne des absences dues à la traditionnelle mobilité de saison sèche, à but économique ou social. S'il est assez courant de sanctionner celui qui s'est absenté afin de poursuivre des activités lucratives, la décision devient plus compliquée si il s'agissait d'assister au baptême d'un neveu. Dans ce cas, payer l'amende équivaut à reconnaître que l'association est contre la tradition, puisque s'absenter à une fête lignagère signifie menacer de rupture les liens tissés dans la communauté locale. Devoirs associatifs et devoirs sociaux entrent donc en conflit.

"Bureaux" et leaders associatifs

Un deuxième clivage dans les associations sépare les responsables des membres qui n'ont pas de rôles spécifiques à jouer. En principe, ce sont les membres fondateurs qui ont un accès privilégié aux postes de responsabilité. Pourtant, le taux de participation formelle des hommes à la gestion des associations reste partout très élevé.

4 En effet, j'ai déjà souligné ailleurs (Blundo, 1991) que les "bureaux" des associations étudiées sont caractérisés par une façade formelle rappelant une organisation bureaucratique, avec sa cohorte de présidents, secrétaires, trésoriers, commissaires aux comptes, contrôleurs de l'irrigation, responsables de la commercialisation et leurs adjoints respectifs.

A ces charges, le plus souvent, ne correspondent pas des tâches précises, et le fonctionnement réel des organisations montre que ces "bureaux" répondent à une double stratégie : légitimer l'association vis-à-vis de l"'extérieur", en lui donnant une image d'organisation "moderne" et efficace, et, à l'intérieur, distribuer, du moins symboliquement, le pouvoir parmi les hommes du groupement, qui partagent le même statut social, et qui sont donc, au sein de l'organisation sociale wolof, des égaux-rivaux (nawle).

6 Toutefois, si cette hypothèse de la distribution symbolique des rôles comme stratégie visant la prévention de conflits d'autorité et de pouvoir est pertinente pour les tâches et les rôles "secondaires", d'ordre technique, les critères d'accès au sommet des associations sont plus complexes.

Je pense en particulier aux figures des trésoriers et des leaders.

Les trésoriers doivent être des personnes d'honnêteté et de moralité éprouvées. Or, ces qualités sont propres, chez les Wolof, aux aînés, aux personnes appartenant à la caste supérieure des géér et à ceux qui jouissent d'un certain prestige. En outre, les trésoriers ne doivent pas avoir de problèmes économiques pouvant les pousser à détourner l'argent du groupe pour les résoudre. 
les règlements - car l'identité et le fonctionnement même des associations sont étroitement liés aux caractéristiques du leader. Si celui-ci est malade, s'il voyage, s'il perd confiance, tout fonctionne à une vitesse réduite. Enfin, s'il abandonne, le groupe se morcelle, comme le montre le cas du groupement de Kasassa, qui a connu quatre ans de crise après la démission de son leader.

ambiguité de la position des leaders des associations paysannes est due au fait que leur légitimité repose partiellement sur la tradition, et que le soutien des membres dépend d'un jeu complexe de facteurs hétérogènes. D'une part, les présidents bénéficient d'un rapport privilégié avec des partenaires externes. Cela se traduit par une augmentation de prestige et d'influence sur la société locale, qui en retour accepte leur rôle d'intermédiaire et leur délègue toute responsabilité. D'autre part, cette légitimité est mise en cause si le leader abuse ouvertement de son pouvoir et surtout, si les résultats attendus de l'engagement collectif tardent à se produire. Le bureau exerce donc un certain contrôle sur le leader désigné, et peut essayer, pour ainsi dire, de le "détrôner" 9 . 
fragilité de la figure du leader.

En juin 1987, l'association de Keur Ngaye doit faire face à une crise aiguë: la campagne maraîchère n'a pas donné les résultats escomptés et un conflit oppose le groupement à des personnages politiquement influents au sein du village. La responsabilité de l'échec retombe sur le président, qui en plus exerce son rôle de façon autoritaire: il garde les clefs du jardin potager, et s'arroge le droit d'en réglementer l'accès des membres du groupement. La grille n'est ouverte qu'aux moments consacrés journellement à l'arrosage et à l'entretien des parcelles. Mais comme une partie du terrain est réservée à l'exploitation individuelle, les maraîchers sont dans l'impossibilité de gérer au mieux leur emploi du temps. Baay Dame, le leader, est suspecté aussi de prélever du secteur communautaire les légumes destinés à la consommation de sa propre famille. Les hommes du "bureau" décident alors de le remplacer. Dans un premier temps, ils essayent de rejeter la responsabilité sur l'ONG d'appui, en demandant à ses dirigeants de destituer Baay Dame. Apprenant la chose, le leader se rend à son tour au siège du COMI pour demander d'être soutenu. Mais les coopérants refusent d'être mêlés à des querelles internes. Le "bureau" convoque alors une réunion générale. A cette occasion, le trésorier, personnage influent dans le village, donne sa démission et propose que le leader en fasse autant, sous le prétexte du renouvellement périodique des fonctions directives de l'association. A la place de Baay Dame, il propose d'élire son vice-président, un captif. Le leader se lève alors et, en dénonçant ce qu'il définit comme un "coup d'État", prononce un discours élogieux de ses qualités et mérites : qui pourrait le remplacer? C'est lui qui le premier démarre le travail, avance toujours l'argent, a même acheté les bancs où les gens sont assis et qui a, en définitive, amené le projet dans le village. Tout le monde se tait et la réunion est levée pour éviter des querelles plus importantes. Après quelques mois, le conflit qui était à l'origine de la "révolte" trouve une solution provisoire. Toutefois, comme le soutien du groupement ne cesse de s'affaiblir, le leader cherchera à tout prix à obtenir de l'ONG d'appui une pompe Gueroult ${ }^{10}$. Elle servira, selon ses dires, "à faire crever d'envie" ses détracteurs et convaincre ses hommes et ses femmes de l'intérêt du "projet".

Baay Dame restera encore en place jusqu'en 1991, année de sa mort, tué par un voisin lors d'une dispute.

Cet exemple met bien en évidence la position ambiguë des leaders, mais aussi le fait que les stratégies des individus font appel à des registres culturels et à des codes d'interaction variés. A bien voir, les stratégies que le "bureau" d'une part, et le leader d'autre part, ont utilisé dans cet affrontement, se rapportent à des logiques différentes, voire opposées.

Si la stratégie initiale - le recours à l'ONG, comme possible source de légitimation, à la fois de la "révolte" et du maintien de l'autorité - est commune aux parties en conflit, ensuite les tactiques divergent : curieusement, les membres du "bureau" invoquent, au nom d'une démocratie douteuse, les règlements associatifs qui, je le répète, ne sont presque jamais appliqués. Ils adoptent donc un discours "moderniste". Le président, en revanche, joue jusqu'au bout le rôle de leader charismatique qui, de temps à autre, accomplit des gestes de générosité et de munificence vis-à-vis du groupement. Toutefois cette attitude, qui est légitime dans un contexte de relations de clientèle, ne peut pas être acceptée dans un groupe de pairs. Il se voit donc obligé de réaffirmer son rôle d'intermédiaire, de courtier, entre son association et l'aide extérieure. L'obtention d'une pompe Gueroult doit être lue dans cette optique.

68 Jusqu'à présent, j'ai décrit les dynamiques internes aux associations. 


\section{Les "associés" et les autres : le conflit à l'échelle villageoise}

71 L'utilisation de l'adjectif "villageois" dans la définition des groupements paysans me semble pertinente dans la mesure où il fait référence à leur dimension territoriale, mais cesse de l'être s'il est employé pour traduire la participation de la communauté villageoise dans son ensemble. En effet, les associations "modernes" étudiées sont souvent calquées sur les clivages qui divisent la société villageoise, et sont donc le monopole de groupes restreints. recrutement concernent l'appartenance au village et l'acceptation des règles et des objectifs du groupement. Toutefois, mesurée à l'échelle villageoise, la représentativité des associations est extrêmement variable : si à Fass Ndjebel, Kasassa et Ndoune plus de la moitié des unités résidentielles est impliquée, au contraire à Keur Ngaye et Nioro Thialène les groupements concernent quelques familles seulement. Et, généralement, les bureaux de chaque association sont formés par des agnats ou par des lignages alliés.

A mieux regarder, dans certains villages les membres sont même "concentrés" dans un espace social déterminé.

L'exemple de l'association de Fass Ndjebel est éclairant: la majorité de ses membres provient des quartiers Callen (34,3\%) et Sise (48,6\%) tandis que dans les deux autres quartiers du village, Ceegen et Ndomen, la participation à l'association est limitée à trois unités résidentielles (kër).

Dans le quartier Callen, six kër participent au groupement: quatre d'entre eux appartiennent au lignage fondateur des Sall et leurs chefs sont donc liés par voie agnatique. Un autre ker descend d'une famille d'esclaves (jaam) de case des Sall; le chef du sixième groupe local de résidence, un Pël Fouta, est aussi lié par des rapports de clientèle au lignage Sall Dans le quartier Sise, l'association voit la participation de six autres kër, appartenant aux lignages $\mathrm{Ba}$, Sise, Toure et Mbaye, liés par voie matrilinéaire et ayant tissé de profonds liens d'alliance par voie d'échanges matrimoniaux.

Qui sont donc les non-associés? Tout d'abord, les minorités ethniques: 96\% des membres des associations appartiennent à l'ethnie majoritaire des Wolof.

Ensuite, les couches de la population situées aux extrêmes de l'échelle économique villageoise. Du point de vue économique, les membres des associations représentent en effet une "classe moyenne" : tant les plus démunis que les plus riches (borom barke) n'en font pas partie.

D'une part, les chefs de famille pauvres sont les plus touchés par l'exode rural saisonnier et désertent donc les activités associatives qui se déroulent surtout durant la saison sèche. D'autre part, les borom barke sont peu concernés et préfèrent observer de loin l'évolution des choses. Pour certains gros éleveurs, les horticulteurs sont même source de problèmes, puisqu'ils exploitent les rares ressources hydriques du village au détriment des animaux. 
Il serait fort intéressant de faire une sociologie des "exclus". Sans vouloir l'entreprendre dans le cadre de cet article, on peut cependant résumer leur point de vue $^{12}$ : il y a ceux qui ont participé dans le passé, mais qui ont quitté les associations à cause de problèmes internes dus à une gestion économique peu claire et au monopole des décisions de la part d'une poignée d'individus; pour d'autres, les activités proposées sont trop contraignantes et mal intégrées avec le calendrier productif traditionnel. Certains affirment aussi avoir été exclus intentionnellement, mais la majorité exprime un sentiment d'ambivalence et d'incertitude à l'égard des groupements : on préfère attendre que les associations évoluent, sans avoir à partager les risques de l'insuccès.

L'existence des groupements paysans n'est certainement pas passée inaperçue dans les villages, surtout depuis qu'ils ont commencé à avoir des relations avec des partenaires extérieurs et qu'ils ont diversifié leurs activités. Durant leurs dix ans de vie, plusieurs conflits ont éclaté entre les cinq groupements et d'autres membres du village.

81 Leurs enjeux différaient selon les cas : dans certains villages, ils étaient principalement de nature foncière. La détention d'un droit d'usage sur une surface déterminée s'avère indispensable pour des associations qui s'adonnent à des activités productrices qui s'étalent sur toute l'année et comportent souvent une mise en valeur durable de la terre. Or, si le droit coutumier ne prévoit pas de cession définitive de terres à une entité non-lignagère, les associations modernes ont aussi un accès difficile au réseau des prêts de terre annuels renouvelables, puisqu'elles nécessitent des terrains sis à des endroits très prisés par les villageois : les champs familiaux (tol kër), situés à proximité des points d'eau, et les champs dans les bas-fonds, en vue d'aménagements hydrauliques parfois importants (micro-barrages, diguettes de retenue des crues, etc.). Ces terrains sont rarement prêtés, a fortiori à une association qui pourrait demander une affectation officielle auprès de la Communauté Rurale suite à une valorisation durable. À titre d'exemple, en 1990, le projet négocié avec l'ONG d'aménager dans les villages les zones de bas-fond avec des micro-barrages a provoqué l'éclatement de litiges fonciers concernant leur emplacement entre les deux groupements de Ndoune et de Keur Ngaye d'une part, et les villageois d'autre part, ce qui a retardé leur réalisation.

Dans d'autres cas, les disputes entre "associés" et "non-associés" concernaient le contrôle de l'aide se déversant sur le village.

83 C'est le cas de l'association de Fass Ndjebel. En 1985, à l'époque des distributions de denrées alimentaires durant la soudure, cette association est accusée par une faction opposée d'avoir accaparé les vivres. L'année suivante, la lutte entre ces deux groupes s'exacerbe: la faction rivale de celle intégrée au projet s'organise elle aussi en groupement de maraîchers et met en place à son tour un jardin, ce qui provoque des tiraillements et de nombreuses défections dans le "camp" adverse.

Mais au-delà des "objets" de litige, ce qui est réellement mis en cause, ce sont les rapports de pouvoir dans les villages. Dans certains cas, des factions préexistantes recourent au modèle associatif comme stratégie et prennent le domaine du développement comme "terrain de combat".

Cela apparaît clairement dans l'exemple donné à l'instant, où les associations qui s'affrontent représentent l'opposition de deux lignages en lutte pour la chefferie. Dans un contexte de lutte entre factions, le contrôle de l'intervention extérieure devient aussi le signe de la suprématie d'un groupe sur l'autre, puisque ses retombées (puits, 
jardin, vivres, moulin à mil) revêtent la double fonction de montrer aux non-associés l'intérêt du "projet" et d'obtenir de la communauté entière la reconnaissance d'être le groupe qui a "amené" quelque chose au village, contrairement au groupe rival. Parfois, les relations entre leaders et membres tournent au clientélisme et les associations fonctionnent alors comme des factions (Blundo, 1991).

Cela apparaît encore plus clairement dans le village de Keur Ngaye, où un conflit, amorcé en 1987 autour d'enjeux initialement de nature foncière, masquait des enjeux économiques et politiques au niveau du village.

Tout a commencé quand l'association s'est opposée à la prétention du chef du village de creuser dans son propre quartier le nouveau puits cofinancé par l'ONG. L'ouvrage a été en revanche réalisé dans un autre quartier, près d'un terrain cultivable cédé par un membre de l'association. Une fois le puits implanté et un verger réalisé, le donateur a décidé inexplicablement de reprendre le terrain. Il avait été sollicité en cela par le chef de village et par le secrétaire de l'association, son allié et membre du Conseil Rural de Koungheul. Il en est résulté un conflit qui est arrivé jusqu'aux bureaux de la Communauté Rurale, et qui, même s'il a été tranché d'autorité par le Sous-préfet, n'a été résolu définitivement qu'après deux ans de négociations.

La pression exercée par le chef de village et le secrétaire du groupement sur le prêteur du terrain pour qu'il revienne sur sa décision est en quelque sorte un moyen pour bloquer des initiatives dont on leur avait refusé le contrôle exclusif. Leur stratégie était d'autant plus payante qu'ils pouvaient faire intervenir leurs réseaux à la Communauté Rurale de Koungheul. Dans cette lutte entre adversaires politiques, semer la confusion dans le groupe rival s'avère être un moyen pour affaiblir la légitimité du leader. En même temps, bloquer les démarches administratives de l'association sert à démontrer à ses membres la puissance de l'autre faction, et donc à réaffirmer leur droit à être les intermédiaires entre l'extérieur et les villageois. Il s'agit probablement d'un conflit né du passage d'un type d'intermédiaire à un autre.

La plupart des conflits, qui ont éclaté apparemment au sein des associations, concernent en réalité d'autres acteurs sociaux villageois, ce qui rend leur résolution plus difficile. Cela n'est pas étonnant, si l'on songe au fait que chaque membre de l'association participe parallèlement à d'autres réseaux et est imbriqué dans d'autres relations d'alliance : ainsi, le prêteur du terrain de Keur Ngaye est un forgeron (tëgg), homme de bas statut et client du chef de village. Il se trouve donc partagé entre le devoir à l'égard de son patron et l'engagement et la parole donnés vis-à-vis de son groupement.

Une variation sur le même thème se présente à Nioro Thialène. La dispute entre hommes et femmes, née à propos de l'utilisation des bénéfices, avait été presque résolue. Mais le conflit s'était élargi à tout le village après l'intervention des maris des femmes associées, qui s'en étaient pris en particulier au chef de village. En réalité, ces hommes, tout en défendant les intérêts de leurs épouses, réaffirmaient leur fidélité à la faction opposée au chef de village et dirigée par son frère agnatique. Le conflit est né du fait que ce dernier était l'aîné de la première épouse mais qu'il était toutefois de quelques années plus jeune que le chef de village, fils de la deuxième épouse. Celui-ci se trouvait être ainsi l'aîné du segment de lignage, mais selon son frère il avait usurpé la chefferie du village. 
91 Ainsi, l'entrée en jeu d'acteurs dont l'opposition s'inscrivait dans une logique factionnelle, a bouleversé la négociation en cours, dont le contrôle a échappé définitivement aux membres de l'association.

Il faut souligner que la lutte entre frères agnatiques pour la succession à la chefferie est inhérente à la structure polygamique de la famille wolof, surtout "lorsque le rang dans l'ordre des naissances est en contradiction avec le rang dans l'ordre de la parenté classificatoire" (Rocheteau, 1975 :6). Il s'agit donc là d'un conflit de type traditionnel, préexistant à l'associationnisme qui a pu s'exprimer dans un espace institutionnel nouveau. Etre leader d'un groupement paysan peut ainsi renforcer une stratégie de légitimation sociale ou politique. Le chef de village de Nioro Thialène, dont le rôle est contesté par une faction politique rivale, cherche à reconquérir le soutien de la base en dirigeant une association intégrée dans un projet de développement.

Conclusion

93 Il y a environ dix ans, Raymond Firth rappelait que la nature réelle de l'anthropologie est d'être "an inquisitive, challenging, uncomfortable discipline, questioning established positions and proclamed values, peering into underlying interests" (1981 : 200). Ainsi, refuser l'image rassurante de paysans mus par les mêmes intérêts et les mêmes contraintes à coopérer pour un but commun, ne relève pas du simple cynisme académique.

En premier lieu, perpétuer l'image historiquement fausse d'une Afrique communautaire peut avoir la fonction politique de "nier les hiérarchies sociales anciennes pour réussir à en imposer des nouvelles" (Amselle, 1988 : 177). Méconnaître les clivages sociaux, ne voir que la dimension de la solidarité dans les villages, n'est sans doute qu'un pas supplémentaire vers la consolidation des groupes occupant le haut de la stratification sociale locale.

95 En deuxième lieu, cette analyse me permet de souligner une fois de plus, s'il en était besoin, que les processus de changement socio-économique sont plus proches d'un modèle conflictuel que d'un modèle harmonieux (Bastide, 1971).

Par ailleurs, l'analyse "par le conflit" permet de mieux cerner la nature et le fonctionnement de ces acteurs sociaux.

97 D'abord, les associations paysannes sont le lieu d'interaction de "groupes stratégiques" distincts, et chaque groupe a son propre cadre de référence quant aux configurations culturelles et aux projets de société. Autrement dit, une pompe Gueroult, dont personne ne nie la fonction évidente d'accès à l'eau, revêtira un sens fort différent selon que l'on adopte le point de vue d'un leader d'association ou celui d'une femme maraîchère. Pour le premier, il peut devenir un instrument de légitimation de son autorité sur le groupe. Pour la deuxième, c'est un moyen d'allégement d'une des plus lourdes tâches féminines.

Ensuite, les groupements étudiés représentent des minorités qui ont su attirer l'aide externe et qui prétendent jouer le rôle d'intermédiaires entre celle-ci et les populations villageoises. Un facteur-clef dans la compréhension de ces associations est donc leurs relations constantes avec des partenaires souvent occidentaux, qui injectent en milieu local de ressources difficiles à obtenir autrement. L'enjeu est de taille : qui saura le premier répondre au défi ? Ceux qui peuvent mobiliser un nombre suffisant d'hommes et de femmes. Mais d'autres groupes - ou factions - existent au niveau villageois, 
comme je l'ai montré. Toute action visant à appuyer ou à collaborer avec ces entités ne peut donc se passer d'une analyse microsociologique préalable.

Quant aux conflits, sont-ils attisés par les groupements "modernes" ou leur préexistent- ils?

Dans la majorité des cas, les antagonismes générés par les associations ne sont pas de nature "nouvelle", mais peuvent être ramenés à des acteurs sociaux traditionnellement en conflit (hommes/femmes, aînés/cadets, parents agnatiques) et à des domaines traditionnellement générateurs de conflit (foncier, pouvoir).

Toutefois, le cadre institutionnel qui sert de toile de fond à ces conflits est sous certains aspects nouveaux, ou tout au moins présente une nature transitoire :

- au niveau organisationnel, il oscille entre le modèle bureaucratique occidental et la distribution des rôles traditionnels ;

- au niveau des formes de participation, il évolue entre un modèle "démocratique" et un principe de fonctionnement fondé sur le consensus et le monopole des décisions par un groupe restreint;

- au niveau des objectifs et des finalités, il s'inscrit dans la tension entre la poursuite de biens et de services d'intérêt collectif et la satisfaction d'intérêts individuels ;

- au niveau du pouvoir, il présente des leaders qui sont intégrés dans la société locale traditionnelle mais sont aussi en quête de légitimités nouvelles à travers le contrôle des flux de l'aide au niveau local.

Les groupements paysans étudiés montrent leur faiblesse moins par le fait d'être le théâtre de conflits, que par leur faible capacité de les résoudre sans recourir à des médiateurs externes (ONG, administration publique). Ce qui leur fait défaut, c'est apparemment cette cohésion, cet esprit de corps tant exaltés par les anthropologues qui étudiaient les fraternités d'âge, les sociétés secrètes et en général, les formes associatives extra-parentales traditionnelles. Leur nom (d'abord Groupements Karikas, maintenant Entente des Groupements du COMI) est sans doute un indicateur de leur nature exogène et de leur dépendance vis-à-vis de l'aide extérieure.

103 Je n'ai pas fait ici l'autopsie des associations de Koungheul: malgré les difficultés rencontrées, elles évoluent et s'adaptent aux situations nouvelles. Si j'ai privilégié dans cette intervention leur dimension conflictuelle, il faut reconnaître que le conflit n'a pas seulement des effets désorganisateurs et désagrégeants. Les crises, comme remarque Bastide, sont souvent le point de départ d'innovations importantes (Bastide, 1971). Il est indéniable que les dures négociations qui ont mis à l'épreuve nos groupements ont donné vie, dans certains cas, à des dynamiques sociales nouvelles dans les villages.

J'aimerais conclure sur une image empruntée encore une fois au village de Keur Ngaye : celle de tout un village rentrant, un soir de l'hivernage 1988, du travail communautaire sur le champ collectif d'arachides réalisé pour la construction de la Grande Mosquée. A mon retour en 1990, ses deux minarets saluent de loin le visiteur, sans que des conflits aient entravé sa réalisation. Et pourtant, c'est le même village que j'ai décrit à l'instant comme déchiré par la lutte des factions. Sur la place principale, les vieux se reposent à l'ombre de la Grande Mosquée. L'écho des projets est lointain. Décidément, le "développement" c'est une autre affaire. 


\section{BIBLIOGRAPHIE}

Amselle, J-L., 1988, "Le développement vu du village", Sociologia Ruralis, vol. XXVIII, n² 2/3, pp. 176- 181.

Bastide, R., 1971, Anthropologie appliquée, Paris, Payot.

Bierschenk, T., 1988, "Development projects as arenas of negociation for strategie groups", Sociologia Ruralis, vol. XXVIII, n. 2/3, pp. 147-160.

Blundo, G., 1990, "Mbootaay : istituzioni associative tradizionali dei Wolof Saalum-saalum (Senegal)", Africa, vol. XLV, n. 3, settembre, pp. 384-409.

1991,"La brique, la terre et le puits : administration locale, factionnalisme et auto-promotion au Sénégal", in Kwan Kai Hong (sous la dir. de ), Jeux et enjeux de l'autopromotion. Vers d'autres formes de coopération au développement, "Cahiers de l'IUED", n²0, IUED-PUF, pp. 103-133.

CER (Centre d'Expansion Rurale), 1988, Plan de développement de la communauté rurale de Koungheul, Secrétariat exécutif des actions des centres d'expansion rurale, s.l.

Diop, A.-B., 1985 La famille wolof, Paris, Karthala.

Dubois, J-P., 1975,"Les Serer et la question des Terres Neuves au Sénégal", Cahiers de l'ORSTOM, sér. Sc. Hum., vol. XII, $n^{\circ}$ l, pp. 81-120.

Firth, R., 1981, "Engagement and detachment : reflection on applying social anthropology to social affairs", Human Organisation, n. 40, pp. 193-201.

Kwan-Kai-Hong, 1991, "Concilier la coopération avec le développement : les perspectives offertes par l'auto-promotion", in Kwan Kaï Hong (sous la dir. de ), Jeux et enjeux de l'autopromotion. Vers d'autres formes de coopération au développement, "Cahiers de l'IUED", n² 20, IUED-PUF, pp. 13-40.

Olivier De Sardan, J.-P., 1990, "Populisme développementiste et populisme en sciences sociales : idéologie, action, connaissance", Cahiers d'Etudes Africaines, 120, XXX 4, pp. 475-492.

Robertson, A.F., 1984,People and State. An anthropology of planned development, Cambridge, Cambridge University Press.

Rocheteau, G., 1975, "Société Wolof et mobilité", Cahiers ORSTOM, sér. Sc. Hum., vol. XII, n¹, pp. 3-18.

Saar, D. et al. 1981, Développement d'une activité maraîchère villageoise dans le Sine-Saloum, Kaolack, ISRA.

\section{NOTES}

1.Rapporté par A. Sylla, La philosophie morale des Wolof, Dakar, Sankoré, 1978, p.98.

2.Mon terrain chez les associations paysannes de l'ex-arrondissement de Koungheul, dans la région de Kaolack (d'avril 1986 à mars 1988, en octobre 1989 et de mai à octobre 1990), s'est déroulé sous le double statut de coopérant et de chercheur, ce qui m'a permis de participer activement à la vie de ces associations et d'être un des acteurs sociaux impliqués dans leur processus de transformation.

3.Source : Centre d'Expansion Rurale (C.E.R.) de Koungheul. 
4. C'est le premier nom que ces associations avaient adopté, en honneur de l'organisation dont elles attendaient le soutien.

5.Le GIE (Groupement d'Intérêt Économique) est une structure régie par la loi n. 84-37 du Il mai 1984. Le GIE, formule qui devrait faciliter l'accès des paysans au crédit, peut être constitué sans capital commun, seuls les membres étant imposables. Les GIE sont immatriculés au Registre du Commerce et du Crédit Immobilier derrier versement d'une somme de 80.000 FCF A pour les frais d'inscription.

6.Pour une analyse récente, voir Blundo, 1990.

7.Parallèlement à cette involution, certains paysans ont compris que, dans la conjoncture économique actuelle, où l'arachide n'est plus rentable, il faut se reconvertir dans d'autres cultures. Alors, ceux-ci s'adonnent entièrement au maraîchage et à l'arboriculture, mais individuellement, en utilisant toutefois les structures collectives gérées par l'association : puits, système d'exhaure de l'eau à traction animale, outils, grillage de protection à coût réduit, etc. Ainsi à Ndoune, village modèle de l'Entente, où le groupement possède 1 ha cultivé de jardin potager et fruitier, deux membres (le président et le secrétaire) ont une production maraîchère et fruitière sur leurs propres champs dépassant de loin celle de l'association.

8.Je parle ici du maraîchage irrigué de saison sèche. Les femmes wolof pratiquaient traditionnellement le maraîchage durant la saison des pluies, sur des petits lopins situés derrière les concessions familiales (ginaw neeg) ou dans les bas-fonds (xuur). Si le maraîchage de saison sèche se diffuse dans la zone de Koungheul au début des années 1970 - sous l'impulsion de l'IRAT, qui avait implanté à Koumbidia une Unité Expérimentale (voir à ce sujet Sarr, 1981) - dans les villages objet d'étude les premières expériences datent du début des années 80 .

9.Ainsi, le leader de l'association de Ndoune a été destitué en 1985 pour avoir détourné, de connivence avec le chef du village, une partie des sacs de vivres délivrés par la mission de Koungheul et destinés aux familles les plus démunies.

10.Système de pompage de l'eau à traction animale.

11.Sans oublier bien sûr, la confrontation avec leurs partenaires extérieurs, potentiels ou effectifs (les organismes d'appui et les services étatiques), fonctionnant selon des logiques parfois divergentes. Mais nous avons limité notre analyse au niveau associatif et villageois.

12.D'après des entretiens sur un échantillon de 29 chefs de famille dans l'ensemble des villages.

\section{AUTEUR}

GIORGIO BLUNDO

Institut Universitaire d'Etudes du Développement (Genève) 